\title{
Two New C21 Steroidal Glycosides from the Roots of Cynanchum paniculatum
}

\author{
Hai-Li Yu ${ }^{1,2} \cdot$ Qin Long ${ }^{1,2} \cdot$ Wen-Fang $\mathrm{Yi}^{1,2} \cdot$ Bao-Jia Yang ${ }^{1,2} \cdot$ Yu Song $^{1,2} \cdot$ Xiao Ding $^{1} \cdot$ Shun-Lin Li ${ }^{1} \cdot$ Xiao-Jiang Hao ${ }^{1}$
}

Received: 27 February 2019 / Accepted: 9 April 2019 / Published online: 26 April 2019

(c) The Author(s) 2019

\section{Abstract}

Two new C21 steroidal glycosides, paniculatumosides H and I, together with four known ones were isolated from the roots of Cynanchum paniculatum (Bge.) Kitag. Their structures were identified by spectroscopic methods including extensive 1D and 2D NMR techniques. All compounds were subjected to detect the anti-tobacco mosaic virus (TMV) activities and their cytotoxities against three human tumor cell lines (SMMC-7721, MDA-MB-231 and A549). The results showed that compounds $\mathbf{1}$ and $\mathbf{5}$ exhibited potent protective activities against TMV, while 2, 4 and $\mathbf{6}$ had moderate effects on the SMMC7721 cancer cells viability.

\section{Graphical Abstract}

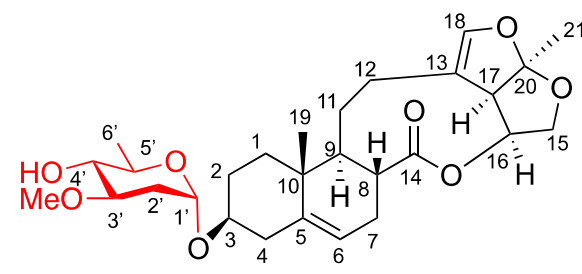

1

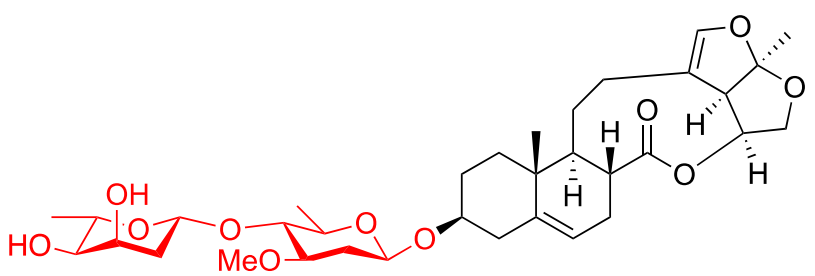

2

Keywords Cynanchum paniculatum $\cdot$ Steroidal glycosides $\cdot$ Bioactivities $\cdot$ NMR data

\section{Introduction}

Cynanchum paniculatum (Bunge) Kitag is a vivacious herb broadly distributed in China, Japan and Korea, whose dried roots have been used as a Chinese herbal medicine for the

\section{Xiao Ding}

dingxiao@mail.kib.ac.cn

$\triangle$ Shun-Lin Li

lisl@mail.kib.ac.cn

1 State Key Laboratory of Phytochemistry and Plant Resources in West China, Kunming Institute of Botany, Chinese Academy of Sciences, Kunming 650201, People's Republic of China

2 University of Chinese Academy of Sciences, Beijing 100049, People's Republic of China treatment of rheumatic arthralgia, epigastric pain, toothache, lumbago, traumatic injuries, and eczema [1-3]. In the previous work, it has been confirmed that $C$. paniculatum contains $\mathrm{C}_{21}$ steroidal glycosides [4, 5]. Pregnanes and their glycosides have shown many aspect activities, such as antitumor, antifungal, antiviral and cytotoxic activities [6, 7]. Our previous works found that pregnanes and their glycosides could inhibit tobacco mosaic virus through suppressing the expression of viral subgenomic RNA but without affecting the accumulation of viral genomic RNA [6]. Therefore, in order to find the structurally unique natural products in C. paniculatum and explore their biological activities, we investigated the dichloromethane extract of $C$. paniculatum, and two new steroidal glycosides paniculatumosides $\mathrm{H}(\mathbf{1})$ and I (2), together with four known ones glaucogenin C (3) [8], cynatratoside A (4) [8], cynapanoside A (5) [9] and 
neocynapanogenin F 3-O- $\beta$-D-oleandropyranoside (6) [10] were isolated. Furthermore, we tested the anti-TMV activities and cytotoxicity of these compounds (Fig. 1).

\section{Result and Discussion}

\subsection{Structure Elucidation}

Paniculatumoside $\mathrm{H}(\mathbf{1})$ was obtained as a yellow powder. Its molecular formula, $\mathrm{C}_{28} \mathrm{H}_{40} \mathrm{O}_{8}$, was determined by analysis the peak at $m / z 527.2629[\mathrm{M}+\mathrm{Na}]^{+}$in the positive HRESIMS (calcd for $\mathrm{C}_{28} \mathrm{H}_{40} \mathrm{O}_{8} \mathrm{Na}$ 527.2615). The IR spectrum displayed absorption bands for hydroxyl $\left(3443 \mathrm{~cm}^{-1}\right)$, carbonyl $\left(1736 \mathrm{~cm}^{-1}\right)$, olefinic $\left(1635 \mathrm{~cm}^{-1}\right)$ and carbon-oxygen bond $\left(1081 \mathrm{~cm}^{-1}\right)$. The ${ }^{1} \mathrm{H}$ NMR spectrum of compound $\mathbf{1}$ showed the presence of two tertiary methyl groups at $\delta_{\mathrm{H}} 0.92(3 \mathrm{H}$, $\mathrm{s}, \mathrm{H}-19)$ and $\delta_{\mathrm{H}} 1.53(3 \mathrm{H}, \mathrm{s}, \mathrm{H}-21)$, one olefinic proton at $\delta_{\mathrm{H}}$ $5.38(\mathrm{~d}, J=5.3 \mathrm{~Hz}, \mathrm{H}-6)$, one olefinic deshielded proton at $\delta_{\mathrm{H}} 6.24$ (s, H-18), two oxygen-substituted methine protons at $\delta_{\mathrm{H}} 3.44(\mathrm{~m}, \mathrm{H}-3)$ and $\delta_{\mathrm{H}} 5.29(\mathrm{td}, 9.5,7.1, \mathrm{H}-16)$ and two methylene protons at $\delta_{\mathrm{H}} 3.84(\mathrm{t}, J=8.6 \mathrm{~Hz}, \mathrm{H}-15 \mathrm{a})$ and $\delta_{\mathrm{H}}$ 4.15 (td, $J=8.6,1.4 \mathrm{~Hz}, \mathrm{H}-15 \mathrm{~b}$ ). By comparison of its ${ }^{1} \mathrm{H}$ and ${ }^{13} \mathrm{C}$ NMR spectra (Table 1) to those of glaucogenin $\mathrm{C}$ (3) indicated that the aglycone of $\mathbf{1}$ is glaucogenin $\mathrm{C}$, which also confirmed by correlations of H-19/H-2b, H-19/H-8, H-1b/H-3, H-21/H-16 and H-16/H-17 in the ROESY experiment (Fig. 2). The main difference was an extra sugar unit present at 1 , which could be further verified by the glycosidation shifts at C-2 (-3.8), C-3 (+4.2) and C-4 (-2.2).
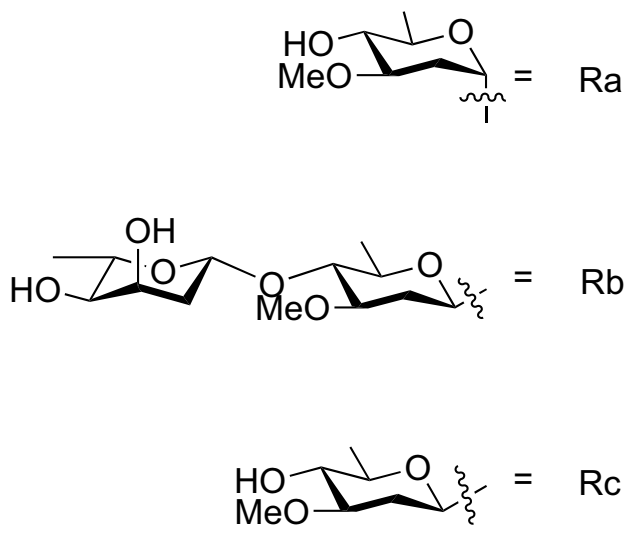

Fig. 1 Chemical structures of compounds 1-6
The sugar carbon signals $\left(\delta_{\mathrm{C}} 95.2,78.2,76.8,67.5,56.5\right.$, $34.5,17.8$ ) illustrated that the sugar belonged to 2,6 -deoxy sugar because it contained four methines, one methylene, a terminal methyl group and a methoxyl group. In the sugar spin systems, the large coupling constants $J_{\mathrm{H}-3^{\prime} / \mathrm{H}-4^{\prime}}(9.1 \mathrm{~Hz})$ and $J_{\mathrm{H}-4^{\prime} / \mathrm{H}-5^{\prime}}(9.3 \mathrm{~Hz})$ disclosed their trans-diaxial relationship, which suggested that the sugar was oleandropyranose [8]. Moreover, the anomeric proton of D-oleandrose was $\alpha$-orientation based on the small coupling constants of $\mathrm{H}-1^{\prime}$ $\left({ }^{3} J_{\mathrm{H}-1, \mathrm{H}-2}=3.5 \mathrm{~Hz}\right)$ [11]. Furthermore, the sugar carbon and proton signals were identical with $\alpha$-D-oleandropyranosyl unit by comparison with reported literature [12], and the ROESY correlations of $\mathrm{H}-2^{\prime} \mathrm{b} / \mathrm{H}-4^{\prime}$ supported this configuration (Fig. 2). Thus, compound 1 could be glycosylated at the C-3 with $\alpha$-linkage by D-oleandropyranosyl unit, which was also concluded from the HMBC correlation of $\delta_{\mathrm{H}} 5.04(\mathrm{~d}$, $J=3.5 \mathrm{~Hz}, \mathrm{H}-1^{\prime}$ ) to $\delta_{\mathrm{C}} 75.8$ (C-3) (Fig. 3). The structure of 1 was finally established as glaucogenin C $3-O-\alpha$-D-oleand ropyranoside.

Paniculatumoside I (2) was obtained as a colourless powder. The molecular formula of $2, \mathrm{C}_{34} \mathrm{H}_{50} \mathrm{O}_{11}$, was deduced by HRESI-MS $\left(\mathrm{m} / z 657.3258[\mathrm{M}+\mathrm{Na}]^{+}\right.$, calcd for $\mathrm{C}_{34} \mathrm{H}_{50} \mathrm{O}_{11} \mathrm{Na}$ 657.3245). The IR spectrum also suggested the presence of hydroxyl $\left(3433 \mathrm{~cm}^{-1}\right)$, carbonyl $\left(1736 \mathrm{~cm}^{-1}\right)$, olefinic $\left(1632 \mathrm{~cm}^{-1}\right)$ and carbon-oxygen bond $\left(1070 \mathrm{~cm}^{-1}\right)$. Compounds 2 and 1 possess the same aglycone by analyzing their ${ }^{1} \mathrm{H}$ and ${ }^{13} \mathrm{C}$ NMR spectra (Table 1), which was confirmed to be glaucogenin $\mathrm{C}$ by detailed analysis of their 2D NMR spectra. Two anomeric proton signals $\delta_{\mathrm{H}} 4.53\left(\mathrm{~d}, J=9.6 \mathrm{~Hz}, \mathrm{H}^{-1}{ }^{\prime}\right)$ and $\delta_{\mathrm{H}} 5.02$

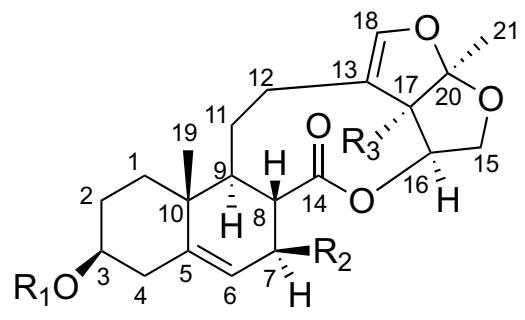

$\begin{array}{cccc}\text { compound } & \mathrm{R}_{1} & \mathrm{R}_{2} & \mathrm{R}_{3} \\ \mathbf{1} & \mathrm{Ra} & \mathrm{H} & \mathrm{H} \\ \mathbf{2} & \mathrm{Rb} & \mathrm{H} & \mathrm{H} \\ \mathbf{3} & \mathrm{H} & \mathrm{H} & \mathrm{H} \\ \mathbf{4} & \mathrm{Rc} & \mathrm{H} & \mathrm{H} \\ \mathbf{5} & \mathrm{Rc} & \mathrm{OH} & \mathrm{H} \\ \mathbf{6} & \mathrm{Rc} & \mathrm{H} & \mathrm{OH}\end{array}$


Table $1{ }^{1} \mathrm{H}$ NMR (500 MHz) and ${ }^{13} \mathrm{C}$ NMR (125 MHz) spectroscopic data for compounds $\mathbf{1}, \mathbf{2}$ and $\mathbf{3}$ in $\mathrm{CDCl}_{3}$

\begin{tabular}{|c|c|c|c|c|c|c|}
\hline \multirow[t]{2}{*}{ No. } & \multicolumn{2}{|l|}{1} & \multicolumn{2}{|l|}{2} & \multicolumn{2}{|l|}{3} \\
\hline & $\delta_{\mathrm{C}}$ & $\delta_{\mathrm{H}}$ & $\delta_{\mathrm{C}}$ & $\delta_{\mathrm{H}}$ & $\delta_{\mathrm{C}}$ & $\delta_{\mathrm{H}}$ \\
\hline $\begin{array}{l}1 \mathrm{a} \\
1 \mathrm{~b}\end{array}$ & 36.2 & $\begin{array}{l}1.96(\mathrm{~m}) \\
1.02(\mathrm{td}, 14.4,3.8)\end{array}$ & 36.4 & $\begin{array}{l}1.96(\mathrm{~m}) \\
1.05(\mathrm{td}, 14.6,3.9)\end{array}$ & 36.4 & $\begin{array}{l}1.96(\mathrm{~m}) \\
1.07(\mathrm{td}, 13.7,3.3)\end{array}$ \\
\hline $\begin{array}{l}2 \mathrm{a} \\
2 \mathrm{~b}\end{array}$ & 27.5 & $\begin{array}{l}1.91(\mathrm{~m}) \\
1.45(\mathrm{~m})\end{array}$ & 29.4 & $\begin{array}{l}1.97(\mathrm{~m}) \\
1.60(\mathrm{~m})\end{array}$ & 31.3 & $\begin{array}{l}1.86(\mathrm{~m}) \\
1.51(\mathrm{~m})\end{array}$ \\
\hline 3 & 75.8 & $3.44(\mathrm{~m})$ & 77.9 & $3.53(\mathrm{~m})$ & 71.6 & $3.53(\mathrm{~m})$ \\
\hline $\begin{array}{l}4 \mathrm{a} \\
4 \mathrm{~b}\end{array}$ & 39.7 & $\begin{array}{l}2.30(\mathrm{~m}) \\
2.24(\mathrm{~m})\end{array}$ & 38.6 & $\begin{array}{l}2.33(\mathrm{~m}) \\
2.16(\mathrm{~m})\end{array}$ & 41.9 & $\begin{array}{l}2.32(\mathrm{dd}, 13.0,2.5) \\
2.17(\mathrm{dd}, 13.0, \\
11.9)\end{array}$ \\
\hline 5 & 140.3 & - & 140.2 & - & 140.2 & - \\
\hline 6 & 120.2 & $5.38(\mathrm{~d}, 5.3)$ & 120.3 & $5.39(\mathrm{~d}, 5.2)$ & 120.2 & $5.39(\mathrm{~d}, 5.3)$ \\
\hline $\begin{array}{l}7 \mathrm{a} \\
7 \mathrm{~b}\end{array}$ & 28.0 & $\begin{array}{l}2.43(\mathrm{~m}) \\
2.05(\mathrm{~m})\end{array}$ & 28.0 & $\begin{array}{l}2.42(\mathrm{~m}) \\
2.04(\mathrm{~m})\end{array}$ & 27.9 & $\begin{array}{l}2.43(\mathrm{~m}) \\
2.02(\mathrm{~m})\end{array}$ \\
\hline 8 & 40.4 & $2.42(\mathrm{~m})$ & 40.4 & $2.42(\mathrm{~m})$ & 40.4 & $2.43(\mathrm{~m})$ \\
\hline 9 & 52.8 & 1.19 (overlapped) & 52.9 & 1.19 (overlapped) & 52.8 & $1.20(\mathrm{t}, 9.7)$ \\
\hline 10 & 38.5 & - & 38.6 & - & 38.3 & - \\
\hline $\begin{array}{l}11 \mathrm{a} \\
11 \mathrm{~b}\end{array}$ & 23.6 & $\begin{array}{l}2.56(\mathrm{~m}) \\
1.29(\mathrm{~m})\end{array}$ & 23.6 & $\begin{array}{l}2.56(\mathrm{~m}) \\
1.28(\mathrm{~m})\end{array}$ & 23.6 & $\begin{array}{l}2.57(\mathrm{~m}) \\
1.27(\mathrm{~m})\end{array}$ \\
\hline $\begin{array}{l}12 \mathrm{a} \\
12 \mathrm{~b}\end{array}$ & 29.6 & $\begin{array}{l}2.06(\mathrm{~m}) \\
1.33(\mathrm{~m})\end{array}$ & 29.6 & $\begin{array}{l}2.07(\mathrm{~m}) \\
1.33(\mathrm{~m})\end{array}$ & 29.6 & $\begin{array}{l}2.07(\mathrm{~m}) \\
1.33(\mathrm{~m})\end{array}$ \\
\hline 13 & 118.1 & - & 118.1 & - & 118.1 & - \\
\hline 14 & 175.6 & - & 175.6 & - & 175.6 & - \\
\hline $\begin{array}{l}15 \mathrm{a} \\
15 \mathrm{~b}\end{array}$ & 67.6 & $\begin{array}{l}4.15(\mathrm{td}, 8.6,1.4) \\
3.84(\mathrm{t}, 8.6)\end{array}$ & 67.6 & $\begin{array}{l}4.15(\mathrm{td}, 7.8,1.6), \\
3.84(\mathrm{t}, 7.8)\end{array}$ & 67.5 & $\begin{array}{l}4.16(\mathrm{td}, 8.1,1.6), \\
3.85(\mathrm{t}, 8.1)\end{array}$ \\
\hline 16 & 75.1 & $5.29(\mathrm{td}, 9.5,7.1)$ & 75.1 & $5.29(\mathrm{td}, 9.7,7.3)$ & 75.0 & $5.29(\mathrm{td}, 9.7,7.8)$ \\
\hline 17 & 55.7 & 3.43 (overlapped) & 55.7 & 3.43 (overlapped) & 55.7 & $3.43(\mathrm{~d}, 7.8)$ \\
\hline 18 & 143.3 & $6.24(\mathrm{~s})$ & 143.3 & $6.25(\mathrm{~s})$ & 143.3 & $6.25(\mathrm{~s})$ \\
\hline 19 & 18.0 & $0.92(\mathrm{~s})$ & 18.0 & $0.91(\mathrm{~s})$ & 18.0 & $0.91(\mathrm{~s})$ \\
\hline 20 & 114.0 & - & 113.9 & - & 113.9 & - \\
\hline 21 & 24.6 & $1.53(\mathrm{~s})$ & 24.6 & $1.53(\mathrm{~s})$ & 24.5 & $1.53(\mathrm{~s})$ \\
\hline \multicolumn{7}{|l|}{ Sugar } \\
\hline $1^{\prime}$ & 95.2 & $5.04(\mathrm{~d}, 3.5)$ & 97.8 & $4.53(\mathrm{~d}, 9.6)$ & & \\
\hline $\begin{array}{l}2^{\prime} \mathrm{a} \\
2^{\prime} \mathrm{b}\end{array}$ & 34.5 & $\begin{array}{l}2.22(\mathrm{~m}) \\
1.50(\mathrm{~m})\end{array}$ & 36.5 & $\begin{array}{l}2.26(\mathrm{~m}) \\
1.53(\mathrm{~m})\end{array}$ & & \\
\hline $3{ }^{\prime} \mathrm{a}$ & 78.2 & $3.52(\mathrm{ddd}, 9.1,8.8,4.9)$ & 79.1 & $3.38(\mathrm{~m})$ & & \\
\hline $4^{\prime}$ & 76.8 & $3.15(\mathrm{dd}, 9.3,9.1)$ & 82.5 & $3.19(\mathrm{dd}, 9.0,8.8)$ & & \\
\hline $5^{\prime}$ & 67.5 & $3.73(\mathrm{dq}, 9.3,6.3)$ & 71.1 & $3.29(\mathrm{~m})$ & & \\
\hline $6^{\prime}$ & 17.8 & $1.28(\mathrm{~d}, 6.3)$ & 18.2 & $1.30(\mathrm{~d}, 6.3)$ & & \\
\hline $3^{\prime}-\mathrm{OCH}_{3}$ & 56.5 & $3.39(\mathrm{~s})$ & 56.4 & $3.40(\mathrm{~s})$ & & \\
\hline $1^{\prime \prime}$ & & & 98.5 & $5.02(\mathrm{~d}, 9.7)$ & & \\
\hline $2^{\prime \prime}$ & & & 38.2 & $\begin{array}{l}2.12(\mathrm{~m}) \\
1.72(\mathrm{~m})\end{array}$ & & \\
\hline $3^{\prime \prime}$ & & & 68.3 & $4.11(\mathrm{~m})$ & & \\
\hline $4^{\prime \prime}$ & & & 72.9 & $3.31(\mathrm{~m})$ & & \\
\hline $5^{\prime \prime}$ & & & 69.6 & $3.74(\mathrm{dq}, 9.7,6.3)$ & & \\
\hline $6^{\prime \prime}$ & & & 18.4 & $1.31(\mathrm{~d}, 6.3)$ & & \\
\hline
\end{tabular}

(d, $J=9.7 \mathrm{~Hz}, \mathrm{H}-1^{\prime \prime}$ ), which correlated to the corresponding anomeric carbon signals at $\delta_{\mathrm{C}} 97.8\left(\mathrm{C}-1^{\prime}\right)$ and 98.5 $\left(\mathrm{C}-1^{\prime \prime}\right)$ in the HSQC spectrum, respectively. It showed that the sugar moiety of $\mathbf{2}$ was made up of two monosaccharides. Meanwhile, from the HSQC, HSQC-TOCSY, and ${ }^{1} \mathrm{H},{ }^{1} \mathrm{H}-\mathrm{COSY}$ experiments, the spin system of each monosaccharide could be established. Two methylenes $\left(\delta_{\mathrm{C}} 36.5\right.$ and 38.2$)$ and methyl groups $\left(\delta_{\mathrm{H}} 1.30\right.$ and 1.31$)$ suggested both monosaccharides of $\mathbf{2}$ were deoxysugars. The splitting patterns and the coupling constants of $\mathrm{H}-1^{\prime}$ 


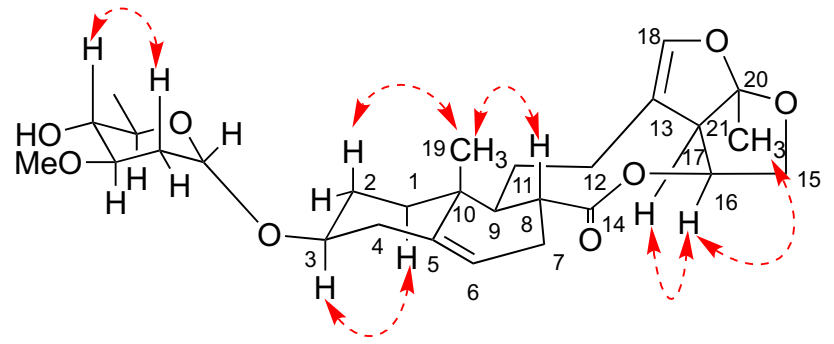

Fig. 2 The key ROESY correlations of $\mathbf{1}$

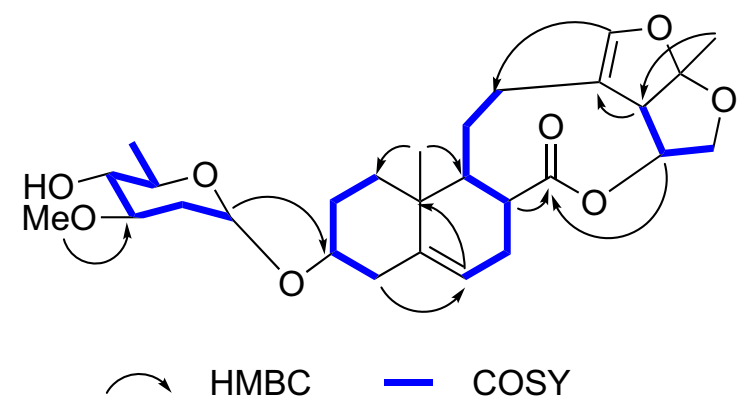

Fig. 3 The selected HMBC and COSY correlations of $\mathbf{1}$

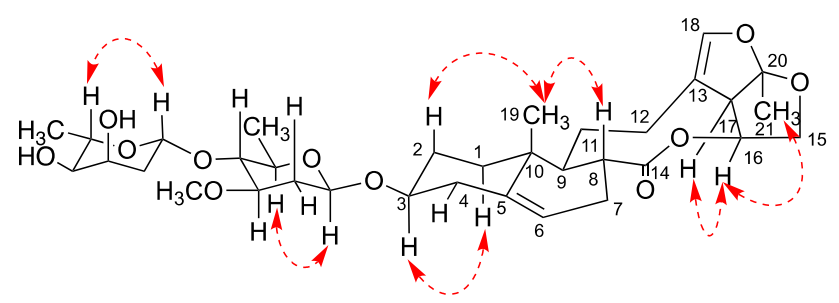

Fig. 4 The key ROESY correlations of 2

$\left({ }^{3} J_{\mathrm{H}-1, \mathrm{H}-2}=9.6 \mathrm{~Hz}\right)$ and $\mathrm{H}-1 "\left({ }^{3} J_{\mathrm{H}-1, \mathrm{H}-2}=9.7 \mathrm{~Hz}\right)$ illustrated that the anomeric protons of the two sugar were $\beta$-oriented, which was also proved by the ROESY collations between $\delta_{\mathrm{H}} 4.53\left(\mathrm{~d}, J=9.6 \mathrm{~Hz}, \mathrm{H}-1^{\prime}\right)$ and $\delta_{\mathrm{H}} 3.29$ $\left(\mathrm{m}, \mathrm{H}-5^{\prime}\right), \delta_{\mathrm{H}} 5.02\left(\mathrm{~d}, J=9.7 \mathrm{~Hz}, \mathrm{H}-1^{\prime \prime}\right)$ and $\delta_{\mathrm{H}} 3.74(\mathrm{dq}$, H-5") (Fig. 4). The oligosaccharide moiety was identified as $\beta$-D-oleandropyranoside and $\beta$-L-digitoxopytanoside by analyzing from comparing the ${ }^{1} \mathrm{H}$ and ${ }^{13} \mathrm{C}$ signals of 2 with those in the literatures [6, 13]. Compound 2 was glycosylated at the $\mathrm{C}-3$, which was concluded from the HMBC correlation of $\delta_{\mathrm{H}} 4.53\left(\mathrm{H}-1^{\prime}\right)$ to $\delta_{\mathrm{C}} 77.9$ $(\mathrm{C}-3)$, and the HMBC correlation of $\delta_{\mathrm{H}} 5.02\left(\mathrm{H}-1^{\prime \prime}\right)$ of $\delta_{\mathrm{C}} 82.5\left(\mathrm{C}-4^{\prime}\right)$ suggested digitoxopytanose connected to $\mathrm{C}-4^{\prime}$ of oleandropyranose (Fig. 5). Therefore, compound 2 was established to be glaucogenin C $3-O-\beta-\mathrm{L}-$ digitoxopytanosyl-( $1 \rightarrow 4)$ - $\beta$-D-oleandropyranoside.

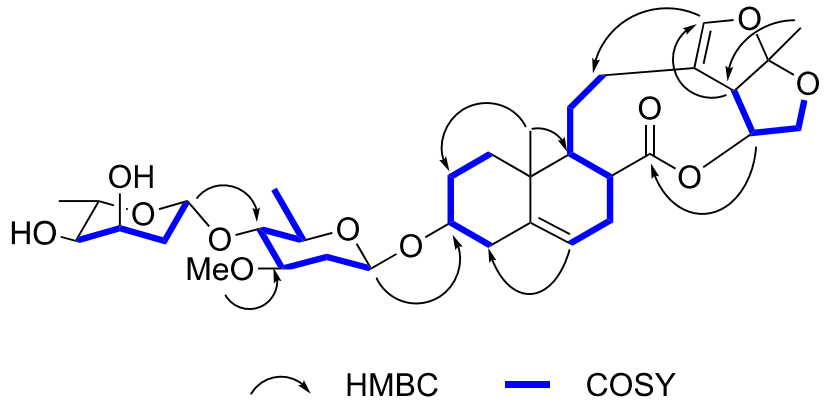

Fig. 5 The selected HMBC and COSY correlations of 2

\subsection{Bioactivities}

\subsubsection{Anti-TMV Activities}

All compounds were tested for anti-TMV activity using the half-leaf method. The results revealed that compounds $\mathbf{1}$ and $\mathbf{5}$ exhibited protective activities at concentration of $200 \mu \mathrm{M}$, with the antiviral inhibition rates of $62.7 \%$ and $59.5 \%$, respectively. Ningnanmycin, which was used as a positive control, showed inhibition rates of $57.3 \%$.

\subsubsection{Cytotoxic Activities}

In order to evaluate whether these compounds have any biological functions on cancer cells, we tested them on SMMC-7721, MDA-MB-231 and A549 human cancer cell lines for their impact on tumor cell growth by MTT method. All the compounds have no inhibitory effect on the MDAMB-231 and A549 cell lines. But compounds 2, 4 and 6 have moderate effects on the SMMC-7721 cancer cells viability with the $\mathrm{IC}_{50}$ values of $27.4 \pm 0.24 \mu \mathrm{M}, 22.2 \pm 0.11 \mu \mathrm{M}$, $27.2 \pm 0.13 \mu \mathrm{M}$, respectively. Cisplatin was used as a positive control and its $\mathrm{IC}_{50}$ for the SMMC-7721 cancer cells was $13.07 \pm 0.27 \mu \mathrm{M}$.

\section{Experimental}

\subsection{General Experimental Procedures}

UV spectra were measured with a Shimadzu UV-2401A spectrophotometer. Optical rotations were determined on a Jasco P-1020 polarimeter. Infrared spectroscopy (IR) spectra were measured on a Bio-Rad FTS-135 spectrometer with $\mathrm{KBr}$ pellets. HRESIMS data were collected on a triple quadrupole mass spectrometer. 1D and 2D NMR spectra were recorded on a Bruker spectrometer with tetramethylsilane as the internal standard. Preparative HPLC separations were carried out using an Agilent 1200 liquid chromatograph with a Waters X-select CSH Prep RP 
C18 $(19 \times 150 \mathrm{~mm})$ column and the flowing rate is $8 \mathrm{~mL} /$ min. Semipreparation HPLC separations were performed on an Agilent 1100 liquid chromatograph using a YMCPack CDS-A $(10 \times 250 \mathrm{~mm})$ column with flowing rate of $3 \mathrm{~mL} / \mathrm{min}$. Sephadex LH-20 (40-70 mm, Amersham Pharmacia Biotech AB, Uppsala, Sweden) and Silica gel (100-200 mesh and 300-400 mesh, Qingdao Marine Chemical, Inc., Qingdao, P. R. China) were used for column chromatography.

\subsection{Plant Material}

The roots of $C$. paniculatum were purchased from a medicinal market (Kunming luosiwan Chinese herbal medicine market) in August 2017 and identified by prof. Hua Peng of Kumming Institute of Botany, Chinese Acdemy of Sciences (CAS).

\subsection{Extraction and Isolation}

The roots of $C$. paniculatum $(100.0 \mathrm{~kg})$ were powdered and extracted three times with $\mathrm{MeOH}$ at room temperature to afford $7.2 \mathrm{~kg}$ of crude extract. The extract was partitioned between $\mathrm{CH}_{2} \mathrm{Cl}_{2}$ and aqueous solution portions which yielded $4.4 \mathrm{~kg}$ crude $\mathrm{CH}_{2} \mathrm{Cl}_{2}$ extract. This extract was subjected to normal-phase silica gel column chromatography eluted with a gradient of petroleum ether-acetone (from 1:0 to $1: 2)$ and $\mathrm{CH}_{2} \mathrm{Cl}_{2}-\mathrm{MeOH}(10: 1-0: 1)$ to obtain eight major fractions (Fr.1-8). Fr.6 (182.3 g) was separated by reversed-phase separation $\left(\mathrm{CH}_{3} \mathrm{OH}-\mathrm{H}_{2} \mathrm{O}, 4: 6-9: 1\right)$ to get twelve subfractions (Fr.6a-61). Fr.6g (2.1 g) was purified by Sephadex LH-20 eluting with $\mathrm{MeOH}$ to yield three fractions. Fr.6g-2 (161.4 mg) was applied to a silica gel column using $\mathrm{CH}_{2} \mathrm{Cl}_{2}-\mathrm{MeOH}(200: 1-0: 1)$ to obtain 3 (7.1 mg). Fr.6g-3 (92.5 mg) was subject to a normal-phase column chromatography and further purified by semiprepative HPLC $(68 \%$ $\mathrm{CH}_{3} \mathrm{CN}$ in water) to yield $6\left(5.0 \mathrm{mg}, \mathrm{t}_{R}=15.0 \mathrm{~min}\right)$. Fr6I $(4.3 \mathrm{~g})$ was separated into four subfractions by Sephadex LH-20 eluting with $\mathrm{CH}_{2} \mathrm{Cl}_{2}-\mathrm{MeOH}$ (1:1). Fr.6I-2 (1.9 g) was chromatographed on a silica gel column eluting with $\mathrm{CH}_{2} \mathrm{Cl}_{2}-\mathrm{MeOH}$ (200:1-0:1) to get Fr.6I-2c (109.2 mg) and Fr.6I-2e (79.3 mg). Fr6I-2c was purified by semiprepative $\mathrm{HPLC}\left(50 \% \mathrm{CH}_{3} \mathrm{CN}\right.$ in water) to obtain 1 (5 mg, $\left.\mathrm{t}_{R}=27.5 \mathrm{~min}\right)$ and $4\left(92.0 \mathrm{mg}, \mathrm{t}_{R}=29.0 \mathrm{~min}\right)$. Fr.6I- $2 \mathrm{~d}$ also used semiprepative HPLC ( $46 \% \mathrm{CH}_{3} \mathrm{CN}$ in water) to yield $5\left(10.2 \mathrm{mg}, \mathrm{t}_{R}=35.0 \mathrm{~min}\right)$. Fr.6 k (3.6 g) was fractioned by a silica gel column eluting with petroleum ether-acetone (80:1-0:1) to afford the Fr6 k-3 (39.3 mg), which was purified by Sephadex LH-20 (MeOH) and semiprepative HPLC (49\% $\mathrm{CH}_{3} \mathrm{CN}$ in water) in sequence to yield 2 (8.2 $\mathrm{mg}$, $\left.\mathrm{t}_{R}=31.5 \mathrm{~min}\right)$.

\subsubsection{Paniculatumoside $\mathrm{H}$ (1)}

White amorphous powder; $[\alpha]_{\mathrm{D}}^{20}-21.5(c 0.11, \mathrm{MeOH})$; IR $(\mathrm{KBr}) \nu_{\max } 3443(\mathrm{OH}), 2919,1736,1635,1384$, $1081 \mathrm{~cm}^{-1}$; ${ }^{1} \mathrm{H}$ and ${ }^{13} \mathrm{C}$ NMR data, see Table 1; ESIMS $m / z 527[\mathrm{M}+\mathrm{Na}]^{+}$; HRESIMS m/z $527.2629[\mathrm{M}+\mathrm{Na}]^{+}$ (calcd for $\mathrm{C}_{28} \mathrm{H}_{40} \mathrm{O}_{8} \mathrm{Na}, 527.2615$ ).

\subsubsection{Paniculatumoside I (2)}

White amorphous powder; $[\alpha]_{\mathrm{D}}^{20}-52.0(c 0.09, \mathrm{MeOH})$; IR $(\mathrm{KBr}) \nu_{\max } 3433(\mathrm{OH}), 2922,1736,1632,1383$, $1070 \mathrm{~cm}^{-1}$; ${ }^{1} \mathrm{H}$ and ${ }^{13} \mathrm{C}$ NMR data, see Table 1; ESIMS $m / z 657[\mathrm{M}+\mathrm{Na}]^{+}$; HRESIMS $m / z 657.3258[\mathrm{M}+\mathrm{Na}]^{+}$ (calcd for $\mathrm{C}_{34} \mathrm{H}_{50} \mathrm{O}_{11} \mathrm{Na}, 657.3245$ ).

\subsection{Biological Activity Assays}

\subsubsection{Anti-TMV Assays}

The half-leaf method was used to evaluate the anti-TMV activities as literatures reported [14]. Ningnanmycin, a Chinese commercial product for plant disease, obtained from Heilongjiang Qiang'er Biochemical Technology Development Company, was administered as a positive control.

\subsubsection{Cytotoxicity Assay}

The cytotoxicity of each compound on three cultured human cancer cell lines was tested by MTT assay. The cell lines used were SMMC-7721 (human hepatoma cells), MDA-MB-231 (triple-negative breast cancer cells), A549 (human lung cancer cell). Cell growth inhibition assay was performed as reported literatures [15]. Cisplatin was used as a positive control.

Acknowledgements This project was supported financially by grants from the National Natural Science Foundation of China (Nos. 31770389, 81703393). We thank Professor Hua Peng, Kunming Institute of Botany (KIB), CAS, for identifying the plant material; Analysis and Test Center, KIB, CAS, for the technical support.

\section{Compliance with Ethical Standards}

Conflicts of interest The authors declare that they have no conflict of interest.

Open Access This article is distributed under the terms of the Creative Commons Attribution 4.0 International License (http://creativeco mmons.org/licenses/by/4.0/), which permits unrestricted use, distribution, and reproduction in any medium, provided you give appropriate credit to the original author(s) and the source, provide a link to the Creative Commons license, and indicate if changes were made. 


\section{References}

1. D. Zhao, B. Feng, S. Chen, G. Chen, Z. Li, X. Lu, X. Sang, X. An, H. Wang, Y. Pei, Fitoterapia 113, 51-57 (2016)

2. S.P. Jiang, Chin J. Chin. Mater. Med. 5, 311-314 (1994)

3. J. Dou, P. Li, Z.M. Bi, J.L. Zhou, Chin. Chem. Lett. 18, 300-302 (2007)

4. H. Mitsuhashi, K. Hayashi, T. Nomura, Chem. Pharm. Bull. 14, 779-783 (1966)

5. K.O. Sugama, K. Hayashi, H. Mitsuhashi, K.O. Kaneko, Chem. Pharm. Bull. 34, 4500-4507 (1986)

6. Y. Yan, J.X. Zhang, K.X. Liu, T. Huang, C. Yan, L.J. Huang, S. Liu, S.Z. Mu, X.J. Hao, Fitoterapia 97, 50-63 (2014)

7. H. Liu, Z. Xiong, F. Li, G. Qu, H. Kobayashi, X. Yao, Chem. Pharm. Bull. 51, 1089-1091 (2003)

8. Z. Zhang, J. Zhou, K. Hayashi, H. Mitsuhashi, Chem. Pharm. Bull. 33, 1507-1514 (1985)
9. K. Sugama, K. Hayashi, H. Mitsuhashi, K. Kaneko, Chem. Pharm. Bull. 34, 4500-4507 (1986)

10. J. Dou, Z.M. Bi, Y.Q. Zhang, P. Li, Chin. J. Nat. Med. 4, 192-194 (2006)

11. J.H. Simpson, Organic Structure Determination Using 2-D NMR Spectroscopy, 2nd edn. (Academic Press, Cambridge, 2011), p. 111

12. X. Huang, A.M. Tan, S.B. Yang, A.Y. Zhang, H. Zhang, Helv. Chim. Acta 92, 937-943 (2009)

13. C. Gui, S. Zhang, X. Zhu, W. Ding, H. Huang, Y.C. Gu, Y. Duan, J. Ju, J. Nat. Prod. 80, 1594-1603 (2017)

14. Z.X. Hu, L.H. Zhao, H.Y. Tang, H.A. Aisa, Y. Zhang, X.J. Hao, Fitoterapia 128, 73-78 (2018)

15. C. Yan, W.Q. Zhang, M. Sun, W. Liang, T.Y. Wang, Y.D. Zhang, X. Ding, Tetrahedron Lett. 59, 4063-4066 (2018) 